\title{
COMPOSICIÓN QUÍMICA Y COMPUESTOS BIOACTIVOS DE TREINTA ACCESIONES DE KIWICHA (Amaranthus caudatus L.)
}

\author{
Ruth Chamorro Gómez ${ }^{* a}$, Ritva Repo Carrasco ${ }^{\mathrm{b}}$, Karina Ccapa Ramírez ${ }^{\mathrm{c}}$, \\ Fredy Quispe Jacobo ${ }^{\mathrm{c}}$
}

\begin{abstract}
RESUMEN
En la presente investigación se evaluaron la composición química y los compuestos bioactivos de 30 accesiones de kiwicha (Amaranthus caudatus L). La fibra dietaria total (FDT) se encontró entre 7,50 a 14,12 \%, los compuestos fenólicos totales entre 51,34 y 75,69 mg GAE/100 g de muestra, los flavonoides totales entre 12,65 a 36,58 mg CE/100 g de muestra. La evaluación de amilosa utilizando como dispersante $\mathrm{NaOH}$ y DMSO presentaron resultados con correlación significativa $\left(r^{2}=0,883\right)$. Se encontraron diferencias estadísticas para todos los compuestos bioactivos y capacidad antioxidante en todas las kiwichas evaluadas. Los análisis estadísticos de correlación de compuestos fenólicos totales con actividad antioxidante de acuerdo al radical 2,2-difenil-1-picrilhidrazilo (DPPH) y 2,2-azinobis (3-etilbenzotiazolina-6-ácido sulfónico) (ABTS) muestran coeficientes de correlación significativas 0,610 y 0,714 , respectivamente. El coeficiente de correlación entre la capacidad antioxidante con el radical DPPH y ABTS fue 0,823 .
\end{abstract}

Palabras clave: ABTS, amilosa, betalaínas, DPPH, fibra dietaria, flavonoides

\section{CHEMICAL COMPOSITION AND BIOACTIVE COMPOUNDS OF THIRTY ACCESSIONS OF KIWICHA (Amaranthus caudatus L.)}

\begin{abstract}
In the present investigation the chemical composition and bioactive compounds of grains of 30 accessions of kiwicha (Amaranthus caudatus L) were evaluated. The total dietary fiber (TDF) was found between 7.50 to $14.12 \%$, the total phenolic compounds between 51.34 to $75.69 \mathrm{mg} \mathrm{GAE} / 100 \mathrm{~g}$ sample, total flavonoids between 12.65 and $36.58 \mathrm{mg} \mathrm{CE} / 100$ $\mathrm{g}$ of sample. The amylose evaluations using $\mathrm{NaOH}$ and DMSO as dispersants showed significant correlation between the results $\left(\mathrm{r}^{2}=0.883\right)$. Statistical differences were found for all the bioactive compounds and antioxidant capacity in all the kiwichas evaluated. Statistical analysis of correlation of total phenolic compounds with antioxidant capacity according to the radical 2,2-diphenyl-1-picrylhydrazyl (DPPH) and 2,2'-azinobis (3-

\footnotetext{
1*a Universidad Nacional Hermilio Valdizán, Av. s/n Universitaria, Huánuco, Perú.

${ }^{\mathrm{b}}$ Universidad Nacional Agraria La Molina, Av. La Molina s/n, La Molina, Lima, Perú.

c Instituto Nacional de Innovación Agraria - INIA. Av. La Molina 1981 Lima, Perú
} 
ethylbenzothiazoline-6-sulfonic acid) (ABTS) showed significant correlation coefficients 0.610 and 0.714 respectively. The correlation coefficient between the antioxidant capacity with the DPPH and ABTS radical was 0.823 .

Key words: ABTS, amylose, betalains, DPPH, dietary fiber, flavonoids

\section{INTRODUCCIÓN}

Kiwicha (Amaranthus caudatus L.) conocido como amaranto, se consumió desde la época precolombina en América del Sur hace miles de años, antes de la incorporación del maíz y la papa en la dieta andina ${ }^{1}$.

Actualmente, este grano andino se revalora por sus propiedades nutricionales y beneficios en la salud al ser consumido como un cereal integral, que crece en condiciones adversas ${ }^{2}$. Estudios recientes destacan su contenido elevado de proteínas ricas en lisina, metionina y cisteína, así como ácidos grasos insaturados, fibra y minerales ${ }^{2}$. Los carbohidratos se encuentran entre 55,5 a $71,5 \%{ }^{1}$.

El conocimiento de la kiwicha como fuente de fitoquímicos se constituye en una fuente potencial de estos compuestos en la dieta humana ${ }^{3}$. Los polifenoles son micronutrientes abundantes en nuestra dieta, a los que se atribuye su rol en la prevención de enfermedades degenerativas tal como cáncer y problemas cardiovasculares, los efectos benéficos de los polifenoles dependen de la cantidad consumida y de su biodisponibilidad ${ }^{4}$.

Las betalaínas son un grupo de aproximadamente 70 pigmentos hidrosolubles, con estructuras de glucósidos, derivados de la 1,7-diazoheptametina y que se han dividido en dos grandes clases: los rojos o betacianinas y los amarillos o betaxantina (figura 1). Un grupo de pigmentos de betalainas tipo betacianinas fueron reportados en la familia Amaranthaceae, las cuales poseen capacidad antioxidante ${ }^{5}$.

A)

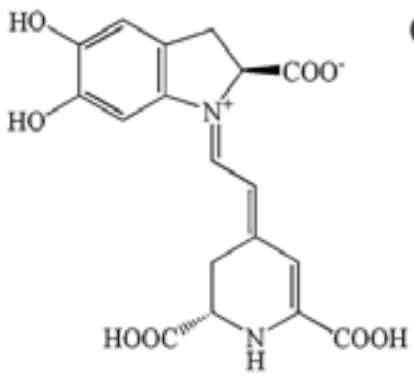

(B)

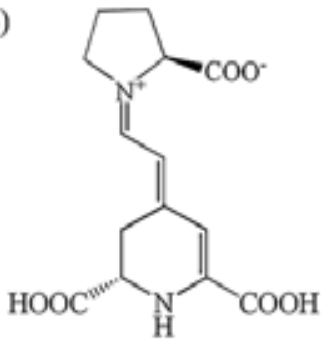

Figura 1. Estructura química de (A) betanidina, una betacianina más común y (B) indicaxantina, una betaxantina ${ }^{5}$. 
La actividad antioxidante de las semillas de kiwicha, evaluada según el método DPPH, en la variedad Centenario presenta 410,0 $\mu \mathrm{mol}$ trolox/g y en la variedad Oscar Blanco 398,1 $\mu \mathrm{mol}$ trolox $/ \mathrm{g}$, mientras que por el método con ABTS valores más altos fueron registrados, $670,1 \mu \mathrm{mol}$ trolox/g en Oscar Blanco y $827,6 \mu \mathrm{mol}$ trolox/g en la variedad Centenario ${ }^{6}$. Investigaciones anteriores reportan actividad antioxidante de compuestos fenólicos totales, flavonoides totales y betalaínas en granos de kiwicha ${ }^{3,6}$.

El consumo de granos andinos aporta fibra a nuestra dieta y ayuda a prevenir las enfermedades cardiovasculares, la fibra insoluble tiene la capacidad de unir las sales biliares en el lumen intestinal, disminuyendo su reabsorción y favoreciendo su eliminación en las deposiciones; para compensar esta pérdida, el hígado resintetiza sales biliares a partir del colesterol del organismo, fenómeno que contribuye a disminuir la colesterolemia. Se ha demostrado que los alimentos ricos en fibra dietética tienden a ser una fuente rica de vitaminas, minerales, fitoquímicos, antioxidantes naturales y otros micronutriente ${ }^{6}$. Los objetivos de la presente investigación fueron evaluar la composición química de 30 accesiones de kiwicha y determinar el contenido de algunos compuestos bioactivos, como fibra dietaria, fenólicos totales, flavonoides totales y betalaínas de 30 accesiones de kiwicha.

\section{PARTE EXPERIMENTAL}

\section{Materiales y reactivos}

30 accesiones de kiwicha de la Estación Experimental Baños del Inca de Cajamarca, Perú, se evaluaron en esta investigación. Los códigos PER de las muestras fueron: PER 002354, PER 002355, PER 002356, PER 002357, PER 002358, PER 002359, PER 002360, PER 002363, PER 002365, PER 002366, PER 002367, PER 002368, PER 002369, PER 002370, PER 002377, PER 002378, PER 002379, PER 002381, PER 002380, PER 002422, PER 002442, PER 002443, PER 002456, PER 002457, PER 002458, PER 002459, PER 002460, PER 002461, PER 002462 y PER 002463. En los análisis se usaron reactivos de las marcas Merck y Sigma Chemical Co, grado analítico.

\section{Métodos}

Composición química. Los contenidos de humedad, ceniza, proteína (factor de conversión $6,25)$, lípidos y fibra cruda fueron determinados según $\mathrm{AOAC}^{7}$. Los carbohidratos se obtuvieron por diferencia.

\footnotetext{
Almidón. La determinación de almidón se realizó según Pérez et al ${ }^{8}$. Los granos de kiwicha se pesaron en tubos de centrífuga de $50 \mathrm{~mL}(2,0 \pm 0,5 \mathrm{~g})$ y se remojaron con agua destilada, toda la noche. Después del tiempo indicado se realizó la molienda por 10 min en una licuadora, y el material triturado se tamizó sobre malla $\mathrm{N}^{\circ} 200(75 \mu \mathrm{m})$. La obtenida se centrifugó a $5000 \mathrm{rpm}$ por $5 \mathrm{~min}$. Se procedió a estandarizar el $\mathrm{pH}=7$. Luego se centrifugó a $5000 \mathrm{rpm}$ por $5 \mathrm{~min}$, se repitió este procedimiento luego de adicionar etanol a $78^{\circ}$ y agua destilada. El sedimentado se secó en una estufa a $40^{\circ} \mathrm{C}$ hasta peso constante ( 48 horas).
} 


\section{Amilosa y Amilopectina}

Hidróxido de sodio $(\mathrm{NaOH})$. La determinación de la amilosa se realizó según Sené et al. ${ }^{9}$. Se pesó $25 \mathrm{mg}$ de almidón en un tubo de prueba de 13 x $100 \mathrm{~mm}$ con tapa roscada y cubierta de teflón. Se incorporó $0,25 \mathrm{~mL}$ de etanol $95^{\circ}$ y mezcló. Seguidamente se adicionó $2,25 \mathrm{~mL}$ de hidróxido de sodio 1 molar, se tapó y mezcló. Se calentó en baño de ebullición por 10 min. Se dejó reposar toda la noche. Entonces se transfirió a una fiola de $25 \mathrm{~mL}$ y se agitó vigorosamente. Para la reacción en un tubo de prueba se incorporó $4 \mathrm{~mL}$ de agua destilada y se midió $0,5 \mathrm{~mL}$ de la muestra. Se adicionó $1 \mathrm{~mL}$ de ácido acético 1 molar y se agitó inmediatamente para adicionar $0,2 \mathrm{~mL}$ de solución de yodo $(0,2 \mathrm{~g}$ de yodo $/ 2 \mathrm{~g}$ yoduro de potasio en $100 \mathrm{~mL}$ ), seguidamente se adicionó $4,3 \mathrm{~mL}$ de agua destilada, se agitó y se dejó reposar por $20 \mathrm{~min}$ en la oscuridad. Se ajustó el espectrofotómetro UV-Vis con el blanco hasta leer cero a $620 \mathrm{~nm}$ y se leyó las soluciones estándar y muestras desconocidas.

Dimetil sulfóxido (DMSO). La determinación de amilosa se realizó según Hoover y Ratnayake ${ }^{10}$. En un vaso precipitado de $10 \mathrm{~mL}$, se pesó $20 \mathrm{mg}$ de almidón y agregó 8 $\mathrm{mL}$ de DMSO al $90 \%$. Esto se ubicó en un agitador magnético con temperatura y agitó vigorosamente. Cuando alcanzó los $85^{\circ} \mathrm{C}$ se controló 15 min. Al término se dejó enfriar el vaso precipitado a temperatura ambiente y diluyó con agua destilada en una fiola volumétrica de $25 \mathrm{~mL}$. En una fiola de $50 \mathrm{~mL}$, se tomó una alícuota de la solución diluida $(1 \mathrm{~mL})$. Se añadió $5 \mathrm{~mL}$ de la solución de yodo $(2 \mathrm{~g}$ de ioduro de potasio/ $/ 2,2 \mathrm{~g}$ de yodo molecular, se enrazó a $500 \mathrm{~mL}$ ). Pronto se enrazó la fiola con agua destilada, mezcló y dejó en reposo durante 15 min en oscuridad (a temperatura ambiente). Se ajustó el espectrofotómetro con el blanco hasta leer cero a $600 \mathrm{~nm}$ y se leyó las soluciones estándar y muestras desconocidas.

Azúcares reductores. Se evaluó según Najmus y Whitney ${ }^{11} ; 0,2 \mathrm{~mL}$ del extracto acuoso de las muestras se mezclaron con $0,8 \mathrm{~mL}$ de la solución ácido dinitrosalicílico ( $30 \mathrm{~g}$ de tartrato de sodio y potasio, $10 \mathrm{~g}$ de ácido dinitrosalićlico y $16 \mathrm{~g}$ de hidróxido de sodio fueron enrazados en $1000 \mathrm{~mL})$ se agitó y se dejó en baño María por $15 \min \left(\sim 100^{\circ} \mathrm{C}\right)$ la muestra fue enfriada inmediatamente en baño de hielo y se agregaron $5 \mathrm{~mL}$ de agua destilada para su evaluación. Al finalizar se midieron las absorbancias a $540 \mathrm{~nm}$ frente a un blanco en el espectrofotómetro UV-Vis. El contenido de azúcares reductores se obtuvo a partir de una curva de calibración de glucosa y expresados como equivalentes $\mathrm{mg}$ de glucosa/100 $\mathrm{g}$ en base seca (bs). Se realizó la curva de calibración de glucosa entre los 250 y $2200 \mathrm{mg} / \mathrm{L}\left(\mathrm{r}^{2}=0,999 ; \mathrm{y}=0,0002 \mathrm{x}-0,0247\right)$.

\section{Compuestos bioactivos}

Fibra dietaria. Se evaluó según el método de la $\mathrm{AACC}^{12}$. Para esto, se pesó $1000 \mathrm{mg}$ de la muestra en un vaso de precipitado de $250 \mathrm{~mL}$ (por cuadruplicado) se adicionó $40 \mathrm{~mL}$ de solución buffer MES-TRIS (19,52 g de 2 ( $N$-morfolino) ácido etanosulfónico (MES) y 14,2 $\mathrm{g}$ de tris (hidroximetil) aminometano (TRIS) en $2 \mathrm{~L}$ de agua destilada, se ajustó el $\mathrm{pH}$ a 8,2 con $\mathrm{NaOH}, 6 \mathrm{~N}$ ), seguido se llevó a cabo una digestión enzimática utilizando $\alpha$-amilosa termoestable $\left(50 \mu \mathrm{L} \mathrm{a} \sim 100^{\circ} \mathrm{C}\right)$, proteasa $\left(100 \mu \mathrm{L}\right.$ a $\left.60{ }^{\circ} \mathrm{C}\right)$ y amiloglusosidasa $(200 \mu \mathrm{L}$ a 60 ${ }^{\circ} \mathrm{C}$ ) para degradar el almidón y las proteínas presentes. El hidrolizado se filtró y el residuo se lavó con $10 \mathrm{~mL}$ de: agua destilada a $70^{\circ} \mathrm{C}$, etanol de $95^{\circ}$ y acetona, luego fue secado y pesado (FDI). El filtrado (FDS) se precipitó con (cuatro volúmenes) etanol de $95^{\circ}$ a $60^{\circ} \mathrm{C}$ ), por $\sim 60$ 
min. Transcurrido el tiempo se lavó con $15 \mathrm{~mL}$ de alcohol de $78^{\circ}, 95^{\circ}$ y acetona, luego se filtró, secó y pesó. A los valores se les restó el contenido de proteína, ceniza y blanco. La fibra dietaria total (FDT) se obtuvo de la suma de la FDI y FDS.

Preparación de la muestra para fenólicos totales, flavonoides totales, capacidad antioxidante y azúcares reductores. La muestra se preparó según Hirose et al. ${ }^{13}$. Se pesó $100 \mathrm{mg}$ de la muestra homogenizada directamente en el tubo de centrífuga de $13 \mathrm{~mL}$ con tapa, se adicionó $10 \mathrm{~mL}$ de etanol:agua destilada $(1: 1)$, se agitó y maceró toda la noche $\left(4^{\circ} \mathrm{C}\right)$. Trascurrido el tiempo, el extracto crudo se filtró a través de un filtro de membrana de $0,45 \mu \mathrm{m}$ y almacenó a $-20^{\circ} \mathrm{C}$ hasta su posterior análisis.

Fenólicos totales. Se utilizó el método adaptado de Hirose et al. ${ }^{13}$. A $3 \mathrm{~mL}$ de agua destilada se agregó $1 \mathrm{~mL}$ del extracto. Y se mezcló en un agitador tipo vórtex. Luego se añadió $1 \mathrm{~mL}$ de reactivo del fenol según Folin-Ciocalteau (1:4). Se homogenizó y finalmente se agregó 1 $\mathrm{mL}$ de carbonato de sodio al $10 \%$. Se mezcló las soluciones por 1 hora, en oscuridad y al término del tiempo indicado se leyó las absorbancias en el espectrofotómetro UV-Vis a 760 $\mathrm{nm}$. El contenido de compuestos fenólicos totales se expresó como mg equivalentes de ácido gálico (GAE)/100 g en base seca. Se realizó la curva de calibración del ácido gálico entre 4 y $40 \mathrm{mg} / \mathrm{L}\left(\mathrm{r}^{2}=0,999 ; \mathrm{y}=0,0166 \mathrm{x}\right)$.

Flavonoides totales. Se determinaron según Dini et al.14. 0,5 mL de extracto se añadió a un tubo de prueba de $10 \mathrm{~mL}$ conteniendo $2 \mathrm{~mL}$ de agua destilada a tiempo cero, se añadieron $0,15 \mathrm{~mL}$ de nitrito de sodio acuoso $(5 \mathrm{~g} / 100 \mathrm{~mL})$. Después de $5 \mathrm{~min}$, se añadieron $0,15 \mathrm{~mL}$ de una solución acuosa de cloruro de aluminio $(10 \mathrm{~g} / 100 \mathrm{~mL})$. A los $6 \mathrm{~min}$, se añadió $1 \mathrm{~mL}$ de hidróxido de sodio 1 molar a la mezcla. Inmediatamente se diluyó al volumen con la adición de 1,2 mL de agua destilada y se agitó hasta que la solución estuvo completamente mezclada. La absorbancia se midió a $510 \mathrm{~nm}$ en el espectrofotómetro UV-Vis frente a un blanco que fue preparado con agua destilada. Los contenidos de flavonoides totales en los extractos se expresaron como mg equivalentes de catequina $(\mathrm{CE}) / 100 \mathrm{~g}$ en base seca. Se realizó la curva de calibración de catequina entre los 10 y $80 \mathrm{mg} / \mathrm{L}\left(\mathrm{r}^{2}=0,998 ; \mathrm{y}=0,0039 \mathrm{x}\right)$.

Betalaínas. Para la determinación de betalaínas, se siguió la metodología de Von Elbe ${ }^{14}$. Se realizó la extracción de las betalaínas de $200 \mathrm{mg}$ de muestra homogenizada con $10 \mathrm{~mL}$ de buffer fosfato a pH 6,5 (4/9,4 (v/v) 8,863 g/L Na $\left.\mathrm{HPO}_{4} / 6,773 \mathrm{~g} / \mathrm{L} \mathrm{KH}_{2} \mathrm{PO}_{4}\right)$ bajo agitación constante a una velocidad media durante 2 horas en oscuridad (a temperatura ambiente). Finalizado el tiempo señalado se centrifugó a $13000 \mathrm{rpm}$ durante $30 \mathrm{~min}$ a $4{ }^{\circ} \mathrm{C}$. Se ajustó el espectrofotómetro con el blanco hasta leer cero a 476, 538 y $600 \mathrm{~nm}$ y se procedió a leer las absorbancias de las muestras desconocidas. La determinación de betacianinas y betaxantinas resultó de reemplazar los valores de absorbancia en las expresiones: $\mathrm{x}=1,095(\mathrm{a}-\mathrm{c}), \mathrm{y}=$ $\mathrm{b}-\mathrm{z}-\mathrm{x} / 3,1$ y $\mathrm{z}=\mathrm{a}-\mathrm{x}$; donde: $\mathrm{a}=$ absorbancia a $538 \mathrm{~nm}, \mathrm{~b}=$ absorbancia a $476 \mathrm{~nm}, \mathrm{c}$ = absorbancia a $600 \mathrm{~nm}, \mathrm{x}=$ absorbancia debido a betacianina, $\mathrm{y}=$ absorbancia debido a betaxantinas, $\mathrm{y} \mathrm{z}=$ absorbancia debido a impurezas. 
Capacidad antioxidante con DPPH. Se determinó según Hirose et al. ${ }^{13} ; 0,3 \mathrm{~mL}$ de extracto se mezcló con 2,7 $\mathrm{mL}$ de solución de DPPH $(0,07 \mathrm{mM})$. La mezcla se agitó vigorosamente y se dejó reposar durante 30 min en oscuridad, la absorbancia se midió a $517 \mathrm{~nm}$ en el espectrofotómetro frente a un blanco. La actividad antioxidante en los extractos se expresó como equivalentes trolox $(\mu \mathrm{mol}) / 100 \mathrm{~g}$ en base seca. Se realizó la curva de calibración de trolox entre los 5 y $47 \mathrm{mg} / \mathrm{L}\left(\mathrm{r}^{2}=0,999 ; \mathrm{y}=0,0008 \mathrm{x}\right)$.

Capacidad antioxidante con ABTS. Se siguió la metodología según Re et al. ${ }^{15}$ se tomó 0,3 mL de extracto y se mezcló con $3 \mathrm{~mL}$ de solución de ABTS (se disolvió ABTS en agua destilada hasta una concentración de $7 \mathrm{mM}$; el radical catión ABTS fue producido haciendo reaccionar la solución de ABTS con 2,45 $\mathrm{mM}$ de persulfato de potasio y dejando permanecer la mezcla en oscuridad a temperatura ambiente durante 12 a 16 horas antes del uso. Debido a que el ABTS y el persulfato de potasio reaccionan estequiométricamente en una proporción de 1:0,5; la absorbancia fue ajustada con etanol a 0,70 $\pm 0,02$ a $734 \mathrm{~nm}$ ). La mezcla se agitó vigorosamente y se dejó reposar durante 6 min en la oscuridad, la absorbancia se midió a $734 \mathrm{~nm}$ en el espectrofotómetro UV-Vis frente a un blanco. La actividad antioxidante en los extractos se expresó como equivalentes trolox $(\mu \mathrm{mol}) / 100 \mathrm{~g}$ en base seca. Se realizó la curva de calibración de trolox entre los 5 y $47 \mathrm{mg} / \mathrm{L}\left(\mathrm{r}^{2}=0,999 ; \mathrm{y}=0,0103 \mathrm{x}\right)$.

Análisis estadístico. Los análisis fueron realizados por triplicado en su mayoría y los resultados se expresaron como medias y su desviación estándar. Los resultados fueron evaluados mediante análisis de varianza (ANVA) y Kruskal-Wallis, según las diferencias entre las accesiones, se consideraron significativas sus diferencias en $\rho \leq 0,05$. Adicionalmente, se realizaron pruebas de significancias según Tukey a $\rho \leq 0,05$. Para establecer la relación entre las diferentes variables se utilizó la correlación No paramétrica de Spearman bivariada a un nivel de significancia de 0,05 y 0,01. Los análisis estadísticos se realizaron en el programa estadístico Statistical Package for the Social Scienses (SPSS) versión 20.

\section{RESULTADOS Y DISCUSIÓN}

Los resultados de la composición química mostraron diferencias significativas en $\rho \leq 0,05$ entre 30 diferentes accesiones de kiwicha, tabla 1. 
Tabla 1. Resultados de la composición química de las accesiones de kiwicha.

\begin{tabular}{|c|c|c|c|c|c|c|c|c|c|c|c|c|c|c|c|c|c|}
\hline \multirow{2}{*}{$\begin{array}{c}\text { Código } \\
\text { Nacional }\end{array}$} & \multicolumn{3}{|c|}{$\begin{array}{c}\text { Humedad } \\
(\%)\end{array}$} & \multicolumn{2}{|c|}{$\begin{array}{l}\text { Ceniza } \\
(\% \text { bs })\end{array}$} & \multicolumn{3}{|c|}{$\begin{array}{l}\text { Lípidos } \\
(\% \text { bs })\end{array}$} & \multicolumn{3}{|c|}{$\begin{array}{c}\text { Proteína } \\
\text { (\% bs) }\end{array}$} & \multicolumn{3}{|c|}{$\begin{array}{c}\text { Fibra cruda } \\
\text { (\% bs) }\end{array}$} & \multicolumn{3}{|c|}{$\begin{array}{c}\text { Carbohidratos } \\
(\% \mathrm{bs})\end{array}$} \\
\hline & 11,30 & \pm & $0,07^{\text {cdefg }}$ & 1,96 & $\pm 0,03^{\circ}$ & 8,13 & \pm & $0,11^{\mathrm{fg}}$ & 15,28 & \pm & $0,02^{\text {efgh }}$ & 2,08 & \pm & $0,02^{\mathrm{jklm}}$ & 74,63 & \pm & $0,08^{\mathrm{abc}}$ \\
\hline PER 002355 & 10,64 & \pm & $0,07^{\mathrm{ijk} k}$ & 2,13 & $\pm 0,05^{\mathrm{n}}$ & 8,72 & \pm & $0,02^{\text {bod }}$ & 15,14 & \pm & $0,08^{\text {ghi }}$ & 2,75 & \pm & $0,05^{\mathrm{bc}}$ & 74,01 & \pm & $0,08^{\text {cdefghtij }}$ \\
\hline PER 002356 & 11,30 & \pm & $0,00^{\text {cdefg }}$ & 2,52 & $\pm 0,02^{\text {ghij }}$ & 8,12 & \pm & $0,09^{\mathrm{fg}}$ & 16,35 & \pm & $0,16^{\mathrm{ab}}$ & 2,34 & \pm & $0,05^{\text {figh }}$ & 73,01 & \pm & $0,06^{\mathrm{k}}$ \\
\hline PER 002357 & 11,07 & \pm & $0,08^{\mathrm{fg}}$ & 2,15 & $\pm 0,04^{\mathrm{n}}$ & 8,38 & \pm & $0,03^{\text {def }}$ & 14,41 & \pm & $0,04^{\mathrm{ij}}$ & 1,90 & \pm & $0,00^{\mathrm{mn}}$ & 75,06 & \pm & $0,10^{\mathrm{ab}}$ \\
\hline PER 002358 & 11,08 & \pm & $0,12^{\mathrm{fg}}$ & 2,64 & $\pm 0,00^{\text {efgh }}$ & 7,91 & \pm & $0,06^{\mathrm{gh}}$ & 15,94 & \pm & $0,14^{\text {abcdef }}$ & 2,56 & \pm & $0,04^{\text {cdef }}$ & 73,52 & \pm & $0,20^{\text {efghijk }}$ \\
\hline PER 002359 & 11,07 & \pm & $0,01^{\mathrm{fg}}$ & 2,50 & $\pm 0,04^{\mathrm{hijk}}$ & 8,20 & \pm & $0,13^{\text {efg }}$ & 15,16 & \pm & $0,12^{\mathrm{fghi}}$ & 2,31 & \pm & $0,04^{\mathrm{ghi}}$ & 74,13 & \pm & $0,21^{\text {cdefg }}$ \\
\hline PER 002360 & 10,67 & \pm & $0,17^{\mathrm{ijk}}$ & 2,72 & $\pm 0,05^{\text {cdef }}$ & 7,89 & \pm & $0,04^{\mathrm{gh}}$ & 15,16 & \pm & $0,11^{\text {ghi }}$ & 1,97 & \pm & $0,01^{\mathrm{klmm}}$ & 74,24 & \pm & $0,05^{\text {cde }}$ \\
\hline PER 002363 & 10,27 & \pm & $0,09^{1}$ & 2,85 & $\pm 0,07^{\mathrm{c}}$ & 8,84 & \pm & $0,11^{\mathrm{bc}}$ & 14,13 & \pm & $0,15^{j}$ & 2,10 & \pm & $0,01^{\mathrm{ijklm}}$ & 74,19 & \pm & $0,25^{\text {cdef }}$ \\
\hline PER 002365 & 10,73 & \pm & $0,04^{\mathrm{hi}}$ & 2,77 & $\pm 0,01^{\mathrm{cde}}$ & 8,54 & \pm & $0,05^{\text {cde }}$ & 15,38 & \pm & $0,12^{\operatorname{defg}}$ & 2,27 & \pm & $0,04^{\text {ghij }}$ & 73,31 & \pm & $0,12^{\mathrm{hijk}}$ \\
\hline PER 002366 & 10,70 & \pm & $0,01^{\mathrm{ij}}$ & 2,37 & $\pm 0,07^{\mathrm{klm}}$ & 8,44 & \pm & $0,16^{\text {def }}$ & 15,62 & \pm & $0,03^{\text {bcdefg }}$ & 2,07 & \pm & $0,03^{\mathrm{jklm}}$ & 73,57 & \pm & $0,19^{\text {defghijk }}$ \\
\hline PER 002367 & 11,49 & \pm & $0,00^{\text {bcd }}$ & 2,33 & $\pm 0,02^{\mathrm{lm}}$ & 7,99 & \pm & $0,10^{\mathrm{gh}}$ & 15,69 & \pm & $0,12^{\text {bcdefg }}$ & 2,44 & \pm & $0,01^{\mathrm{efg}}$ & 73,99 & \pm & $0,04^{\text {cdefghij }}$ \\
\hline PER 002368 & 11,11 & \pm & $0,07^{\mathrm{fg}}$ & 2,32 & $\pm 0,02^{\mathrm{lm}}$ & 8,47 & \pm & $0,06^{\text {cdef }}$ & 16,18 & \pm & $0,16^{\text {abc }}$ & 2,47 & \pm & $0,02^{\text {defg }}$ & 73,03 & \pm & $0,00^{k}$ \\
\hline PER 002369 & 11,22 & \pm & $0,07^{\text {defg }}$ & 2,58 & $\pm 0,01^{\text {fghi }}$ & 8,40 & \pm & $0,02^{\text {def }}$ & 15,75 & \pm & $0,00^{\text {bcdefg }}$ & 2,44 & \pm & $0,00^{\text {efg }}$ & 73,26 & \pm & $0,05^{\mathrm{ijk}}$ \\
\hline PER 002370 & 11,43 & \pm & $0,03^{\text {bcde }}$ & 2,31 & $\pm 0,04^{\mathrm{lm}}$ & 6,88 & \pm & $0,08^{k}$ & 15,55 & \pm & $0,03^{\text {cdefg }}$ & 2,14 & \pm & $0,04^{\text {hijkl }}$ & 75,26 & \pm & $0,05^{\mathrm{a}}$ \\
\hline PER 002377 & 11,08 & \pm & $0,01^{\mathrm{fg}}$ & 2,79 & $\pm 0,02^{\text {cd }}$ & 7,24 & \pm & $0,07^{\mathrm{jk}}$ & 15,50 & \pm & $0,01^{\text {cdefg }}$ & 2,01 & \pm & $0,07^{\mathrm{klm}}$ & 74,46 & \pm & $0,06^{\text {abc }}$ \\
\hline PER 002378 & 11,26 & \pm & $0,01^{\text {cdefg }}$ & 2,23 & $\pm 0,01^{\mathrm{mn}}$ & 7,40 & \pm & $0,16^{\mathrm{ij}}$ & 16,01 & \pm & $0,16^{\text {abcde }}$ & 1,97 & \pm & $0,00^{\mathrm{klmm}}$ & 74,35 & \pm & $0,32^{\text {bed }}$ \\
\hline PER 002379 & 11,04 & \pm & $0,00^{\mathrm{gh}}$ & 2,22 & $\pm 0,01^{\mathrm{mn}}$ & 8,13 & \pm & $0,03^{\mathrm{fg}}$ & 15,52 & \pm & $0,01^{\text {cdefg }}$ & 2,69 & \pm & $0,07^{\text {cd }}$ & 74,12 & \pm & $0,02^{\text {cdefg }}$ \\
\hline PER 002381 & 11,36 & \pm & $0,03^{\text {cdef }}$ & 3,67 & $\pm 0,02^{\mathrm{a}}$ & 7,66 & \pm & $0,01^{\mathrm{hi}}$ & 15,28 & \pm & $0,11^{\text {efgh }}$ & 5,79 & \pm & $0,13^{\mathrm{a}}$ & 73,38 & \pm & $0,13^{\text {frhijk }}$ \\
\hline PER 002380 & 11,85 & \pm & $0,01^{\mathrm{a}}$ & 2,79 & $\pm 0,01^{\text {cd }}$ & 8,57 & \pm & $0,02^{\text {cde }}$ & 16,59 & \pm & $0,00^{\mathrm{a}}$ & 6,00 & \pm & $0,12^{\mathrm{a}}$ & 72,04 & \pm & $0,01^{1}$ \\
\hline PER 002422 & 11,69 & \pm & $0,01^{\mathrm{ab}}$ & 1,96 & $\pm 0,01^{\circ}$ & 7,68 & \pm & $0,06^{\mathrm{hi}}$ & 16,11 & \pm & $0,14^{\text {abcd }}$ & 1,92 & \pm & $0,03^{\operatorname{lmn}}$ & 74,26 & \pm & $0,17^{\text {cde }}$ \\
\hline PER 002442 & 11,57 & \pm & $0,01^{\mathrm{abc}}$ & 2,65 & $\pm 0,00^{\operatorname{def} g}$ & 8,54 & \pm & $0,12^{\text {cde }}$ & 15,80 & \pm & $0,02^{\text {bedefg }}$ & 2,73 & \pm & $0,07^{\mathrm{bc}}$ & 73,01 & \pm & $0,11^{\mathrm{k}}$ \\
\hline PER 002443 & 11,03 & \pm & $0,00^{\mathrm{gh}}$ & 2,41 & $\pm 0,00^{\mathrm{jkl}}$ & 7,91 & \pm & $0,20^{\mathrm{gh}}$ & 16,17 & \pm & $0,13^{\text {abc }}$ & 2,12 & \pm & $0,01^{\mathrm{ijklm}}$ & 73,52 & \pm & $0,07^{\text {efghijk }}$ \\
\hline PER 002456 & 11,13 & \pm & $0,02^{\text {efg }}$ & 2,43 & $\pm 0,01^{j k l}$ & 9,67 & \pm & $0,02^{\mathrm{a}}$ & 14,53 & \pm & $0,01^{\mathrm{hij}}$ & 2,04 & \pm & $0,00^{\mathrm{klm}}$ & 73,37 & \pm & $0,03^{\text {ghijk }}$ \\
\hline PER 002457 & 11,10 & \pm & $0,04^{\mathrm{fg}}$ & 2,33 & $\pm 0,03^{\mathrm{lm}}$ & 9,09 & \pm & $0,02^{\mathrm{b}}$ & 15,37 & \pm & $0,07^{\text {defg }}$ & 2,94 & \pm & $0,04^{\mathrm{b}}$ & 73,21 & \pm & $0,16^{\mathrm{jk}}$ \\
\hline PER 002458 & 10,61 & \pm & $0,11^{\mathrm{ijk} k}$ & 2,74 & $\pm 0,01^{\mathrm{cde}}$ & 7,92 & \pm & $0,07^{\text {gh }}$ & 15,25 & \pm & $0,01^{\text {efgh }}$ & 2,17 & \pm & $0,06^{\text {hijk }}$ & 74,09 & \pm & $0,02^{\text {cdefgh }}$ \\
\hline PER 002459 & 10,18 & \pm & $0,02^{1}$ & 2,44 & $\pm 0,02^{\mathrm{ijkl}}$ & 7,99 & \pm & $0,01^{\mathrm{gh}}$ & 15,52 & \pm & $0,04^{\text {cdefg }}$ & 2,00 & \pm & $0,04^{\mathrm{klm}}$ & 74,05 & \pm & $0,06^{\text {cdefghi }}$ \\
\hline PER 002460 & 10,39 & \pm & $0,18^{j \mathrm{kl}}$ & 2,35 & $\pm 0,01^{\mathrm{klm}}$ & 7,63 & \pm & $0,01^{\text {hi }}$ & 15,67 & \pm & $0,09^{\text {bcdeff }}$ & 1,76 & \pm & $0,00^{\mathrm{n}}$ & 74,34 & \pm & $0,10^{\text {bcd }}$ \\
\hline PER 002461 & 10,38 & \pm & $0,16^{\mathrm{kl}}$ & 3,00 & $\pm 0,08^{\mathrm{b}}$ & 8,43 & \pm & $0,08^{\text {def }}$ & 15,31 & \pm & $0,10^{\text {efg }}$ & 2,48 & \pm & $0,02^{\text {defg }}$ & 73,26 & \pm & $0,10^{\mathrm{ijk}}$ \\
\hline PER 002462 & 10,48 & \pm & $0,11^{\mathrm{ijkl}}$ & 2,10 & $\pm 0,00^{\mathrm{no}}$ & 7,46 & \pm & $0,01^{\mathrm{ij}}$ & 15,88 & \pm & $0,13^{\text {abcdefg }}$ & 2,04 & \pm & $0,03^{\mathrm{klm}}$ & 74,56 & \pm & $0,22^{\text {abc }}$ \\
\hline PER 002463 & 10,71 & \pm & $0,08^{\mathrm{i}}$ & 2,66 & $\pm 0,01^{\text {defg }}$ & 7,00 & \pm & $0,12^{\mathrm{k}}$ & 15,77 & \pm & $0,77^{\text {bcdefg }}$ & 2,60 & \pm & $0,04^{\text {cde }}$ & 74,58 & \pm & $0,57^{\text {abc }}$ \\
\hline
\end{tabular}

En la tabla 1 se observa altos contenidos de proteínas (PER 002380, PER 002356, PER 002368, PER 002443, PER 002422, PER 002378, PER 002358 y PER 002462 con 16,59; 16,$35 ; 16,18 ; 16,17 ; 16,11 ; 16,01 ; 15,94$ y $15,88 \%$ bs, respectivamente, estas accesiones son estadísticamente similares) y lípidos (hasta 9,67 \% la PER 002456, estadísticamente superior).

Los granos de kiwicha contenían almidón entre 53,47 a 63,58 \%, correspondientes a los códigos PER 002381 y PER 002462, respectivamente (tabla 2). La correlación entre almidón y carbohidratos fue significativa con $r=0,497$ a 0,01 y con azúcares reductores $(0,424)$. Pérez et al. ${ }^{8}$ desarrollaron una metodología para la obtención de almidón el cual requiere el remojado de granos, tamizado luego de la molienda, estandarización de $\mathrm{pH}$ y centrifugado con etanol. Método que es propio de este grano debido a que presenta alto contenido de grasa, proteína y fibra; en comparación, por ejemplo, con un tubérculo, pues la extracción de almidón es más sencilla y no requiere de las operaciones antes mencionadas. En investigaciones anteriores ${ }^{16}$ reportan contenidos ligeramente superiores. Otros autore ${ }^{17}$ reportan dentro de lo encontrado en esta investigación.

Los granos de kiwicha contenían amilosa (con $\mathrm{NaOH}$ ) entre 0,36 a 16,60 \% (tabla 2). Correspondientes a las semillas con código PER 002370 y PER 002378, respectivamente. Mientras que la amilopectina se encontraba presente entre 83,40 a 99,64\%, propios de los códigos PER 002378 y PER 002370, respectivamente. Los granos de kiwicha contenían 
amilosa (con DMSO) entre 1,77 a 16,74 \% pertenecientes a los códigos nacionales PER 002356 y PER 002378, respectivamente. Por su parte la amilopectina se encontró entre 83,26 a 98,23 \%; propios de la PER 002378 y PER 002356. Cabe resaltar que los resultados usando ambas metodologías arrojan valores similares que muestran que la PER 002378, presenta mayor contenido de amilosa (16,74\% con DMSO y $16,60 \%$ con $\mathrm{NaOH})$, esto lo confirma el coeficiente de correlación Spearman $r=0,881$; el cual resultó significativa al nivel 0,01 . El criterio tomado en esta investigación para usar $600 \mathrm{~nm}$ para DMSO y $620 \mathrm{~nm}$ para $\mathrm{NaOH}$, fue hacer un barrido y apreciar la mayor absorbancia, luego de la formación del complejo yodo y amilosa, a las longitudes mencionadas correspondientes a cada metodología. En investigaciones realizadas emplearon ${ }^{17}$ enzimas para determinar la amilosa estimando con el ratio de glucosa oxidasa-peroxidasa, con lecturas a $510 \mathrm{~nm}$,

En la tabla 2, los azúcares reductores se encontraron entre 1,60 a 4,51 \% expresado como glucosa, pertenecientes a las semillas con códigos PER 002365 y PER 002462. En una investigación ${ }^{18}$ se evaluaron el efecto de la germinación en el contenido de azúcares reductores expresados en sucrosa, encontraron que el grano de kiwicha sin germinar tenía $0,26 \%$ de sucrosa y conforme se incrementaban las horas de germinación también incrementaban los azúcares reductores alcanzando hasta $11,7 \%$ al evaluar el grano germinado por 20 horas.

Tabla 2. Resultados de almidón, amilosa y amilopectina (con DMSO y NaOH) y azúcares reductores

\begin{tabular}{|c|c|c|c|c|c|c|c|c|c|c|c|c|c|c|c|c|c|c|}
\hline \multirow{2}{*}{$\begin{array}{c}\begin{array}{c}\text { Código } \\
\text { Nacional }\end{array} \\
\text { PER } 002354\end{array}$} & \multicolumn{3}{|c|}{ Almidón (\%) } & \multicolumn{3}{|c|}{$\begin{array}{c}\text { Amilosa } \\
\text { DMSO (\%) }\end{array}$} & \multicolumn{3}{|c|}{$\begin{array}{c}\text { Amilopectina DMSO } \\
(\%)\end{array}$} & \multicolumn{3}{|c|}{$\begin{array}{c}\text { Amilosa } \\
\mathrm{NaOH}(\%)\end{array}$} & \multicolumn{3}{|c|}{$\begin{array}{l}\text { Amilopectina } \\
\mathrm{NaOH}(\%)\end{array}$} & \multicolumn{3}{|c|}{$\begin{array}{l}\text { Azúcares reductores } \\
\text { (g de glucosa/100 g } \\
\text { bs) }\end{array}$} \\
\hline & 56,37 & \pm & $0,59^{\text {hijklm }}$ & 3,05 & \pm & $0,00^{\mathrm{mn}}$ & 96,95 & \pm & $0,00^{\mathrm{ef}}$ & 2,94 & \pm & $0,10^{k}$ & 97,06 & \pm & $0,10^{f}$ & 2,58 & \pm & $0,10^{\text {efghi }}$ \\
\hline PER 002355 & 56,84 & \pm & $0,82^{\text {hijklm }}$ & 2,32 & \pm & $0,00^{\text {opqr }}$ & 97,68 & \pm & $0,00^{\text {abcod }}$ & 1,62 & \pm & $0,21^{\mathrm{lm}}$ & 98,38 & \pm & $0,21^{\mathrm{de}}$ & 2,45 & \pm & $0,03^{\text {efghi }}$ \\
\hline PER 002356 & 56,22 & \pm & $0,86^{\mathrm{ijk} k \mathrm{~m}}$ & 1,77 & \pm & $0,12^{\mathrm{r}}$ & 98,23 & \pm & $0,12^{\mathrm{a}}$ & 1,43 & \pm & $0,04^{\operatorname{lm} n}$ & 98,57 & \pm & $0,04^{\text {cde }}$ & 2,43 & \pm & $0,14^{\text {efghij }}$ \\
\hline PER 002357 & 56,75 & \pm & $0,38^{\text {hijklm }}$ & 2,56 & \pm & $0,00^{\text {nopq }}$ & 97,44 & \pm & $0,03^{\text {bede }}$ & 1,39 & \pm & $0,18^{\operatorname{lmn}}$ & 98,61 & \pm & $0,18^{\text {cde }}$ & 2,19 & \pm & $0,13^{\text {ghijkl }}$ \\
\hline PER 002358 & 54,77 & \pm & $0,46^{\mathrm{Imn}}$ & 2,26 & \pm & $0,12^{\text {opqr }}$ & 97,74 & \pm & $0,12^{\text {abcd }}$ & 1,29 & \pm & $0,18^{\mathrm{mn}}$ & 98,71 & \pm & $0,18^{\mathrm{cd}}$ & 2,52 & \pm & $0,36^{\text {efghi }}$ \\
\hline PER 002359 & 57,89 & \pm & $0,22^{\text {efghij }}$ & 2,74 & \pm & $0,12^{\text {nop }}$ & 97,26 & \pm & $0,12^{\mathrm{cde}}$ & 0,75 & \pm & $0,05^{\mathrm{op}}$ & 99,25 & \pm & $0,05^{\mathrm{ab}}$ & 1,74 & \pm & $0,09^{\mathrm{jkl}}$ \\
\hline PER 002360 & 58,72 & \pm & $0,23^{\text {cdefgh }}$ & 2,07 & \pm & $0,00^{\mathrm{pqr}}$ & 97,93 & \pm & $0,00^{\mathrm{abc}}$ & 0,77 & \pm & $0,16^{\mathrm{op}}$ & 99,23 & \pm & $0,16^{\mathrm{ab}}$ & 2,70 & \pm & $0,40^{\text {cdefgh }}$ \\
\hline PER 002363 & 54,80 & \pm & $0,84^{\mathrm{klmn}}$ & 1,95 & \pm & $0,14^{\mathrm{qr}}$ & 98,05 & \pm & $0,14^{\mathrm{ab}}$ & 1,54 & \pm & $0,40^{\mathrm{lm}}$ & 98,46 & \pm & $0,40^{\mathrm{de}}$ & 2,15 & \pm & $0,34^{\text {ghijkl }}$ \\
\hline PER 002365 & 56,89 & \pm & $0,82^{\text {ghijklm }}$ & 2,87 & \pm & $0,12^{\mathrm{mno}}$ & 97,13 & \pm & $0,12^{\text {def }}$ & 1,79 & \pm & $0,12^{1}$ & 98,21 & \pm & $0,12^{\mathrm{e}}$ & 1,60 & \pm & $0,02^{1}$ \\
\hline PER 002366 & 57,28 & \pm & $0,49^{\text {fhhij }}$ & 13,11 & \pm & $0,37^{\mathrm{b}}$ & 86,89 & \pm & $0,37^{9}$ & 12,43 & \pm & $0,20^{\mathrm{b}}$ & 87,57 & \pm & $0,20^{\circ}$ & 2,00 & \pm & $0,05^{\mathrm{hijkl}}$ \\
\hline PER 002367 & 55,70 & \pm & $0,46^{\mathrm{jlmn}}$ & 12,32 & \pm & $0,49^{\text {cd }}$ & 87,68 & \pm & $0,49^{\text {op }}$ & 11,74 & \pm & $0,22^{\mathrm{c}}$ & 88,26 & \pm & $0,22^{\mathrm{n}}$ & 1,70 & \pm & $0,09^{\mathrm{kl}}$ \\
\hline PER 002368 & 56,33 & \pm & $0,92^{\text {hijklm }}$ & 11,16 & \pm & $0,12^{\mathrm{fg}}$ & 88,84 & \pm & $0,12^{\mathrm{lm}}$ & 10,30 & \pm & $0,17^{\mathrm{e}}$ & 89,70 & \pm & $0,17^{1}$ & 2,04 & \pm & $0,06^{\mathrm{hijkl}}$ \\
\hline PER 002369 & 56,30 & \pm & $0,69^{\text {hijklm }}$ & 5,79 & \pm & $0,37^{1}$ & 94,21 & \pm & $0,37^{\mathrm{g}}$ & 3,83 & \pm & $0,04^{j}$ & 96,17 & \pm & $0,04^{\mathrm{g}}$ & 2,20 & \pm & $0,13^{\text {ghijkl }}$ \\
\hline PER 002370 & 57,15 & \pm & $0,68^{\text {fyhijkl }}$ & 3,05 & \pm & $0,28^{\mathrm{mn}}$ & 96,95 & \pm & $0,28^{\mathrm{ef}}$ & 0,36 & \pm & $0,04^{\mathrm{p}}$ & 99,64 & \pm & $0,04^{\mathrm{a}}$ & 2,65 & \pm & $0,22^{\text {defgh }}$ \\
\hline PER 002377 & 60,35 & \pm & $0,14^{\text {bcde }}$ & 3,56 & \pm & $0,48^{\mathrm{m}}$ & 96,44 & \pm & $0,48^{\mathrm{f}}$ & 1,08 & \pm & $0,05^{\mathrm{no}}$ & 98,92 & \pm & $0,05^{\mathrm{bc}}$ & 2,84 & \pm & $0,10^{\text {cdefg }}$ \\
\hline PER 002378 & 58,39 & \pm & $0,14^{\text {defghi }}$ & 16,74 & \pm & $0,12^{\mathrm{a}}$ & 83,26 & \pm & $0,39^{r}$ & 16,60 & \pm & $0,08^{\mathrm{a}}$ & 83,40 & \pm & $0,08^{\mathrm{p}}$ & 3,39 & \pm & $0,29^{\mathrm{bc}}$ \\
\hline PER 002379 & 58,11 & \pm & $0,54^{\text {efghij }}$ & 12,66 & \pm & $0,40^{\mathrm{bc}}$ & 87,34 & \pm & $0,40^{\mathrm{pq}}$ & 11,04 & \pm & $0,48^{\mathrm{d}}$ & 88,96 & \pm & $0,48^{\mathrm{m}}$ & 2,53 & \pm & $0,08^{\text {efghi }}$ \\
\hline PER 002381 & 53,47 & \pm & $0,90^{\mathrm{n}}$ & 10,89 & \pm & $0,23^{\mathrm{fgh}}$ & 89,11 & \pm & $0,23^{\mathrm{klm}}$ & 10,11 & \pm & $0,04^{\mathrm{ef}}$ & 89,89 & \pm & $0,04^{\mathrm{kl}}$ & 1,90 & \pm & $0,05^{\mathrm{ijkl}}$ \\
\hline PER 002380 & 54,58 & \pm & $0,33^{\mathrm{mn}}$ & 10,34 & \pm & $0,24^{\mathrm{h}}$ & 89,66 & \pm & $0,24^{\mathrm{k}}$ & 9,84 & \pm & $0,05^{\mathrm{f}}$ & 90,16 & \pm & $0,05^{\mathrm{k}}$ & 2,22 & \pm & $0,04^{\text {ghijkl }}$ \\
\hline PER 002422 & 59,46 & \pm & $0,82^{\text {bcdef }}$ & 10,34 & \pm & $0,65^{\mathrm{h}}$ & 89,66 & \pm & $0,65^{\mathrm{k}}$ & 9,88 & \pm & $0,12^{\text {ef }}$ & 90,12 & \pm & $0,12^{\mathrm{kl}}$ & 2,64 & \pm & $0,03^{\text {defgh }}$ \\
\hline PER 002442 & 54,48 & \pm & $0,84^{\mathrm{mn}}$ & 11,44 & \pm & $0,28^{\text {ef }}$ & 88,56 & \pm & $0,28^{\mathrm{mn}}$ & 7,80 & \pm & $0,04^{\mathrm{i}}$ & 92,20 & \pm & $0,04^{\mathrm{h}}$ & 3,29 & \pm & $0,10^{\text {bcd }}$ \\
\hline PER 002443 & 59,54 & \pm & $0,62^{\text {bcdef }}$ & 6,99 & \pm & $0,12^{\mathrm{k}}$ & 93,01 & \pm & $0,12^{\mathrm{h}}$ & 7,51 & \pm & $0,12^{\mathrm{i}}$ & 92,49 & \pm & $0,12^{\mathrm{h}}$ & 2,37 & \pm & $0,09^{\text {fyhijk }}$ \\
\hline PER 002456 & 59,30 & \pm & $0,25^{\text {bedefg }}$ & 10,52 & \pm & $0,12^{\text {gh }}$ & 89,48 & \pm & $0,12^{\mathrm{kl}}$ & 11,00 & \pm & $0,05^{\mathrm{d}}$ & 89,00 & \pm & $0,05^{\mathrm{m}}$ & 2,50 & \pm & $0,10^{\text {efghi }}$ \\
\hline PER 002457 & 60,74 & \pm & $0,77^{\text {bcd }}$ & 9,61 & \pm & $0,14^{\mathrm{i}}$ & 90,39 & \pm & $0,14^{j}$ & 9,24 & \pm & $0,04^{\mathrm{h}}$ & 90,76 & \pm & $0,04^{\mathrm{i}}$ & 2,67 & \pm & $0,11^{\text {defgh }}$ \\
\hline PER 002458 & 61,02 & \pm & $0,01^{\mathrm{bc}}$ & 10,40 & \pm & $0,12^{\mathrm{h}}$ & 89,60 & \pm & $0,12^{k}$ & 9,10 & \pm & $0,14^{\mathrm{h}}$ & 90,90 & \pm & $0,14^{\mathrm{i}}$ & 3,56 & \pm & $0,22^{\mathrm{b}}$ \\
\hline PER 002459 & 61,34 & \pm & $0,36^{\mathrm{ab}}$ & 11,87 & \pm & $0,23^{\mathrm{de}}$ & 88,13 & \pm & $0,23^{\text {no }}$ & 9,18 & \pm & $0,08^{\mathrm{h}}$ & 90,82 & \pm & $0,08^{\mathrm{i}}$ & 2,28 & \pm & $0,05^{\text {fghijkl }}$ \\
\hline PER 002460 & 57,66 & \pm & $0,84^{\text {fghij }}$ & 11,13 & \pm & $0,12^{\mathrm{fg}}$ & 88,87 & \pm & $0,12^{\operatorname{lm}}$ & 11,08 & \pm & $0,08^{\mathrm{d}}$ & 88,92 & \pm & $0,08^{\mathrm{m}}$ & 2,97 & \pm & $0,02^{\text {bedef }}$ \\
\hline PER 002461 & 57,25 & \pm & $0,52^{\text {ffhijk }}$ & 8,70 & \pm & $0,23^{j}$ & 91,30 & \pm & $0,23^{\mathrm{i}}$ & 9,70 & \pm & $0,04^{\mathrm{fg}}$ & 90,30 & \pm & $0,04^{\mathrm{jk}}$ & 3,09 & \pm & $0,77^{\text {bcde }}$ \\
\hline PER 002462 & 63,58 & \pm & $0,27^{\mathrm{a}}$ & 9,43 & \pm & $0,12^{\mathrm{i}}$ & 90,57 & \pm & $0,12^{j}$ & 9,29 & \pm & $0,04^{\mathrm{gh}}$ & 90,71 & \pm & $0,04^{\mathrm{ij}}$ & 4,51 & \pm & $0,80^{\mathrm{a}}$ \\
\hline PER 002463 & 63,56 & \pm & $0,09^{\mathrm{a}}$ & 12,11 & \pm & $0,23^{\text {cde }}$ & 87,89 & \pm & $0,23^{\text {nop }}$ & 11,35 & \pm & $0,10^{\text {cd }}$ & 88,65 & \pm & $0,10^{\mathrm{mn}}$ & 2,34 & \pm & $0,04^{\mathrm{fghijk}}$ \\
\hline
\end{tabular}


En los resultados de la fibra dietaria (tabla 3), las semillas del código PER 002380 alcanzaron un máximo de $14,12 \%$ de FDT, siendo la PER 002422 con 7,50 \% la accesión con menor contenido de fibra dietaria. Según lo esperado la FDI se encontró en mayor proporción con un rango de variación de 6,11 \% (PER 002422) hasta 13,04 \% (PER 002380). Por otro lado, la FDS fluctuaba entre $0,52 \%$ (PER 002456) a 1,97\% (PER 002356).

Los resultados de fibra dietaria revelan que el $88 \%$ en promedio de la FDT fue insoluble y el $12 \%$ en promedio fue soluble (tabla 3 ). La correlación entre FDI y FDT presentó una relación significativa con $\mathrm{r}=0,929$ al nivel 0,01 . Por su parte la correlación entre FDS $\mathrm{y}$ amilopectina con NAOH fue significativa con $r=0,603$ al nivel 0,01. Investigaciones anteriores 14 en fibra dietaria en granos de kiwicha reportan valores similares a los hallados en las semillas evaluadas. Mientras que otros autores reportan contenidos inferiores ${ }^{1}$ y que la FDI está compuesta por ácido galacturónico $(28,5 \%)$, arabinosa $(15,3 \%)$, xilosa $(9,9 \%)$, glucosa $(21,7 \%)$, galactosa $(6,7 \%)$, ramnosa $(6,5 \%)$, glucosa (proveniente de celulosa; $5,9 \%)$, fucosa $(2,3 \%)$ y mannosa $(3,2 \%)$. Mientras que la FDS se encuentra compuesta por glucosa $(24,5 \%)$, ácido galacturónico $(38,6 \%)$, arabinosa $(15,6 \%)$, galactosa $(7,3 \%)$, mannosa $(6,9 \%)$ y xilosa $(2,7 \%)$.

Tabla 3. Resultados de Fibra dietaria insoluble, soluble y total de 30 accesiones evaluadas.

\begin{tabular}{|c|c|c|c|c|c|c|c|c|c|}
\hline $\begin{array}{l}\text { Código } \\
\text { Nacional }\end{array}$ & \multicolumn{3}{|c|}{ FDI (\%) } & \multicolumn{3}{|r|}{ FDS $(\%)$} & & & FDT $(\%)$ \\
\hline PER 002354 & 10,48 & \pm & $0,56^{\text {bc }}$ & 0,99 & \pm & $0,19^{\text {ghijklm }}$ & 11,47 & \pm & $0,51^{\text {bcd }}$ \\
\hline PER 002355 & 8,21 & \pm & $0,33^{\text {defghi }}$ & 0,93 & \pm & $0,15^{\text {hijklm }}$ & 9,14 & \pm & $0,30^{\text {efghi }}$ \\
\hline PER 002356 & 9,80 & \pm & $1,01^{\mathrm{bcd}}$ & 1,97 & \pm & $0,38^{\mathrm{a}}$ & 11,78 & \pm & $1,30^{\mathrm{bc}}$ \\
\hline PER 002357 & 8,47 & \pm & $0,80^{\text {cdefg }}$ & 1,67 & \pm & $0,29^{\text {abcdef }}$ & 10,14 & \pm & $0,80^{\text {cdef }}$ \\
\hline PER 002358 & 7,94 & \pm & $0,27^{\text {defghij }}$ & 1,33 & \pm & $0,32^{\text {bcdefghijkl }}$ & 9,27 & \pm & $0,08^{\text {efghi }}$ \\
\hline PER 002359 & 7,52 & \pm & $0,49^{\text {efghij }}$ & 1,84 & \pm & $0,01^{\text {abcd }}$ & 9,37 & \pm & $0,50^{\text {defghi }}$ \\
\hline PER 002360 & 10,97 & \pm & $0,34^{\mathrm{b}}$ & 1,66 & \pm & $0,35^{\text {abcdef }}$ & 12,63 & \pm & $0,67^{\mathrm{ab}}$ \\
\hline PER 002363 & 8,03 & \pm & $1,02^{\text {defghij }}$ & 1,46 & \pm & $0,19^{\text {abcdefghi }}$ & 9,50 & \pm & $1,17^{\text {detghi }}$ \\
\hline PER 002365 & 7,87 & \pm & $0,54^{\text {defghij }}$ & 1,20 & \pm & $0,38^{\text {efghijkl }}$ & 9,07 & \pm & $0,42^{\text {efghi }}$ \\
\hline PER 002366 & 7,49 & \pm & $0,98^{\text {efghij }}$ & 0,85 & \pm & $0,18^{\text {hijklm }}$ & 8,34 & \pm & $1,15^{\text {fghi }}$ \\
\hline PER 002367 & 7,74 & \pm & $0,81^{\text {efghij }}$ & 0,76 & \pm & $0,08^{\mathrm{klm}}$ & 8,50 & \pm & $0,81^{\text {efghi }}$ \\
\hline PER 002368 & 9,01 & \pm & $0,92^{\text {bcdef }}$ & 0,73 & \pm & $0,22^{\mathrm{lm}}$ & 9,75 & \pm & $0,91^{\text {cdefgh }}$ \\
\hline PER 002369 & 6,49 & \pm & $0,50^{\text {ghij }}$ & 1,26 & \pm & $0,18^{\text {defghijkl }}$ & 7,74 & \pm & $0,35^{\mathrm{hi}}$ \\
\hline PER 002370 & 7,76 & \pm & $0,73^{\text {efghij }}$ & 1,35 & \pm & $0,26^{\text {abcdefghijkl }}$ & 9,11 & \pm & $0,90^{\text {efghi }}$ \\
\hline PER 002377 & 9,01 & \pm & $0,76^{\text {bcdef }}$ & 1,62 & \pm & $0,30^{\text {abcdefg }}$ & 10,63 & \pm & $0,80^{\text {bcde }}$ \\
\hline PER 002378 & 7,01 & \pm & $0,32^{\text {fohij }}$ & 0,91 & \pm & $0,16^{\text {hijklm }}$ & 7,92 & \pm & $0,18^{\text {ghi }}$ \\
\hline PER 002379 & 8,24 & \pm & $0,21^{\text {defghi }}$ & 0,82 & \pm & $0,09^{\mathrm{jklm}}$ & 9,05 & \pm & $0,29^{\text {efghi }}$ \\
\hline PER 002381 & 10,77 & \pm & $0,48^{\mathrm{b}}$ & 0,99 & \pm & $0,15^{\text {ghijklm }}$ & 11,75 & \pm & $0,35^{\mathrm{bc}}$ \\
\hline PER 002380 & 13,04 & \pm & $0,35^{\mathrm{a}}$ & 1,08 & \pm & $0,11^{\text {efghijklm }}$ & 14,12 & \pm & $0,42^{\mathrm{a}}$ \\
\hline PER 002422 & 6,11 & \pm & $0,28^{\mathrm{j}}$ & 1,39 & \pm & $0,15^{\text {abcdefghijk }}$ & 7,50 & \pm & $0,23^{\mathrm{i}}$ \\
\hline PER 002442 & 7,82 & \pm & $0,41^{\text {defghij }}$ & 1,16 & \pm & $0,26^{\text {efghijklm }}$ & 8,98 & \pm & $0,45^{\text {efghi }}$ \\
\hline PER 002443 & 7,96 & \pm & $0,99^{\text {defghij }}$ & 1,04 & \pm & $0,27^{\text {fghijklm }}$ & 9,00 & \pm & $1,10^{\text {efghi }}$ \\
\hline PER 002456 & 8,17 & \pm & $1,18^{\text {defghi }}$ & 0,52 & \pm & $0,16^{\mathrm{m}}$ & 8,69 & \pm & $1,34^{\text {efghi }}$ \\
\hline PER 002457 & 7,11 & \pm & $0,75^{\text {fghij }}$ & 1,41 & \pm & $0,21^{\text {abcdefghij }}$ & 8,51 & \pm & $0,63^{\text {efghi }}$ \\
\hline PER 002458 & 8,30 & \pm & $1,12^{\text {defgh }}$ & 1,72 & \pm & $0,38^{\text {abcde }}$ & 10,02 & \pm & $1,04^{\text {cdefg }}$ \\
\hline PER 002459 & 6,23 & \pm & $1,37^{\mathrm{ij}}$ & 1,48 & \pm & $0,05^{\text {abcdefgh }}$ & 7,71 & \pm & $1,33^{\mathrm{hi}}$ \\
\hline PER 002460 & 9,37 & \pm & $0,77^{\text {bcde }}$ & 0,82 & \pm & $0,21^{\mathrm{ijklm}}$ & 10,19 & \pm & $0,74^{\text {cdef }}$ \\
\hline PER 002461 & 7,11 & \pm & $0,62^{\text {fghij }}$ & 1,90 & \pm & $0,31^{\mathrm{abc}}$ & 9,01 & \pm & $0,76^{\text {efghi }}$ \\
\hline PER 002462 & 8,23 & \pm & $0,56^{\text {defghi }}$ & 1,95 & \pm & $0,19^{\mathrm{ab}}$ & 10,19 & \pm & $1,82^{\text {cdef }}$ \\
\hline
\end{tabular}


En los resultados de compuestos bioactivos (tabla 4), la accesión PER 002363 tuvo menor contenido de compuestos fenólicos totales, mientras que la PER 002380 presentó mayor contenido. Al respecto contenidos superiores a los encontrados en 30 accesiones de semillas evaluadas se reportaron ${ }^{6}$. También se hallaron valores por debajo de lo encontrado en esta investigación ${ }^{1}$. Investigadores cultivaron 18 genotipos de Amaranthus paralelamente en Argentina, México, España y República Checa; llegando a la conclusión de que el contenido de polifenoles en las semillas fue muy influenciada por los factores medioambientales y la especie. Asimismo, encontraron que la A. hypochondriacus presentaba mayor contenido ${ }^{19}$. Se encontró relación positiva (ejemplo: a mayor concentración de fenólicos totales, mayor capacidad antioxidante con DPPH) entre: fenólicos totales y capacidad antioxidante con DPPH; fenólicos totales y capacidad antioxidante con ABTS; flavonoides totales y capacidad antioxidante con DPPH y flavonoides totales y capacidad antioxidante con ABTS con coeficiente de correlación de 0,$610 ; 0,714 ; 0,292$ y 0,385 .

De la tabla 4, se puede notar que la PER 002369 tuvo un menor contenido de flavonoides totales con 12,65 mg CE/100 g bs, mientras que la kiwicha con código PER 002380 presentó el mayor contenido con $36,58 \mathrm{mg} \mathrm{CE} / 100 \mathrm{~g}$ bs. Investigadores ${ }^{19}$ evaluaron el contenido de flavonoides totales en granos de kiwicha, después de cultivar en diferentes condiciones medio ambientales a 18 genotipos de Amaranthus. Encontraron que flavonoides como la rutina, exhibieron grandes variaciones, sin embargo, la nicoticlorin fue menos afectada.

En las semillas con códigos PER 002355, PER 002356, PER 002367, PER 002370, PER 002378, PER 002442, PER 002443, PER 002456, PER 002458 y PER 002460, no se detectaron betalaínas; sin embargo, la PER 002359 alcanzó un máximo de 1,01 mg/100 $\mathrm{g}$ bs. Hubo diferencias significativas entre todas las accesiones. Contenidos superiores de betalaínas en semillas de kiwicha fueron reportados, entre 50 a 199 mg/100 g 20.

Al evaluar la reacción entre los compuestos con actividad antioxidante y el radical estable 2,2-difenil-1-picrilhidrazilo (DPPH), se encontró un rango entre 119,15 a 268,20 $\mu \mathrm{mol} \mathrm{TE} / 100$ $\mathrm{g}$ bs, los códigos correspondientes fueron PER 002354 y PER 002380, respectivamente (tabla 4). La reducción de color del radical ABTS por el efecto de un antioxidante se pudo evaluar leyendo la absorbancia a $734 \mathrm{~nm}$ y la decoloración del color violeta del radical DPPH a 517 nm. 
Tabla 4. Compuestos bioactivos de las accesiones de kiwicha.

\begin{tabular}{|c|c|c|c|c|c|c|c|c|c|c|c|c|c|c|c|}
\hline \multirow{3}{*}{$\begin{array}{c}\begin{array}{c}\text { Código } \\
\text { Nacional }\end{array} \\
\text { PER } 002354\end{array}$} & \multirow{2}{*}{\multicolumn{3}{|c|}{$\begin{array}{c}\text { Fenólicos totales (mg } \\
\text { GAE } / 100 \mathrm{~g} \mathrm{bs})\end{array}$}} & \multirow{2}{*}{\multicolumn{3}{|c|}{$\begin{array}{c}\text { Flavonoides totales } \\
\text { (mg CE/100 g bs) }\end{array}$}} & \multirow{2}{*}{\multicolumn{3}{|c|}{$\begin{array}{l}\text { Betalaínas (mg/100 g } \\
\text { bs) }\end{array}$}} & \multicolumn{3}{|c|}{$\begin{array}{l}\text { Actividad Antioxidante } \\
\text { según DPPH }\end{array}$} & \multicolumn{3}{|c|}{$\begin{array}{c}\text { Actividad Antioxidante } \\
\text { según ABTS }\end{array}$} \\
\hline & & & & & & & & & & \multicolumn{6}{|c|}{$(\mu \mathrm{mol} \mathrm{TE} / 100 \mathrm{~g} \mathrm{bs})$} \\
\hline & 52,75 & \pm & $0,50^{\mathrm{mno}}$ & 30,99 & \pm & $0,87^{\mathrm{c}}$ & 0,82 & \pm & $0,00^{\mathrm{c}}$ & 119,15 & \pm & $7,43^{\mathrm{p}}$ & 456,71 & \pm & $8,49^{\text {no }}$ \\
\hline PER 002355 & 67,24 & \pm & $0,73^{\text {cd }}$ & 35,11 & \pm & $0,87^{\mathrm{ab}}$ & 0,00 & \pm & $0,00^{\text {ad }}$ & 161,37 & \pm & $11,35^{\text {defghi }}$ & 654,70 & \pm & $10,74^{\circ}$ \\
\hline PER 002356 & 63,74 & \pm & $1,45^{\text {ghi }}$ & 27,54 & \pm & $0,80^{\mathrm{d}}$ & 0,00 & \pm & $0,00^{a c}$ & 173,28 & \pm & $3,19^{\text {bcd }}$ & 598,09 & \pm & $14,54^{\mathrm{ef}}$ \\
\hline PER 002357 & 66,87 & \pm & $0,93^{\text {cd }}$ & 30,38 & \pm & $0,83^{\mathrm{c}}$ & 0,56 & \pm & $0,00^{\mathrm{g}}$ & 169,67 & \pm & $5,35^{\text {bcde }}$ & 667,85 & \pm & $7,60^{\mathrm{bc}}$ \\
\hline PER 002358 & 65,16 & \pm & $0,47^{\text {efg }}$ & 22,71 & \pm & $0,04^{\mathrm{ij}}$ & 0,82 & \pm & $0,00^{\mathrm{f}}$ & 153,33 & \pm & $2,48^{\text {ghijk }}$ & 565,26 & \pm & $7,72^{\text {ghi }}$ \\
\hline PER 002359 & 54,27 & \pm & $0,42^{\mathrm{lm}}$ & 33,88 & \pm & $0,74^{\mathrm{b}}$ & 1,01 & \pm & $0,00^{\mathrm{a}}$ & 125,44 & \pm & $2,97^{\text {nop }}$ & 476,39 & \pm & $3,84^{\mathrm{n}}$ \\
\hline PER 002360 & 62,26 & \pm & $0,60^{\mathrm{i}}$ & 18,22 & \pm & $0,86^{\mathrm{m}}$ & 0,55 & \pm & $0,00^{\mathrm{h}}$ & 146,40 & \pm & $5,57^{\mathrm{ijkl}}$ & 542,06 & \pm & $2,06^{\mathrm{jkl}}$ \\
\hline PER 002363 & 51,34 & \pm & $0,43^{\circ}$ & 19,79 & \pm & $0,81^{\mathrm{klm}}$ & 0,07 & \pm & $0,00^{\mathrm{t}}$ & 121,97 & \pm & $2,23^{\mathrm{op}}$ & 453,32 & \pm & $6,44^{\circ}$ \\
\hline PER 002365 & 58,00 & \pm & $0,17^{\mathrm{k}}$ & 25,17 & \pm & $0,89^{\text {efg }}$ & 0,29 & \pm & $0,00^{\mathrm{k}}$ & 124,85 & \pm & $0,47^{\text {nop }}$ & 533,37 & \pm & $6,64^{\mathrm{kl}}$ \\
\hline PER 002366 & 62,20 & \pm & $1,02^{\mathrm{i}}$ & 21,33 & \pm & $0,85^{\mathrm{jkl}}$ & 0,37 & \pm & $0,00^{\mathrm{i}}$ & 120,88 & \pm & $2,20^{\text {op }}$ & 523,95 & \pm & $5,11^{1}$ \\
\hline PER 002367 & 64,56 & \pm & $0,28^{\mathrm{fgh}}$ & 23,26 & \pm & $0,87^{\text {ghij }}$ & 0,00 & \pm & $0,00^{a b}$ & 161,83 & \pm & $5,34^{\text {defgh }}$ & 558,10 & \pm & $6,99^{\text {hij }}$ \\
\hline PER 002368 & 67,55 & \pm & $0,42^{\mathrm{c}}$ & 25,03 & \pm & $0,93^{\text {fgh }}$ & 0,86 & \pm & $0,00^{\circ}$ & 169,20 & \pm & $10,57^{\text {bodef }}$ & 630,36 & \pm & $2,86^{\mathrm{d}}$ \\
\hline PER 002369 & 64,28 & \pm & $0,35^{\mathrm{gh}}$ & 12,65 & \pm & $0,86^{\mathrm{n}}$ & 0,90 & \pm & $0,00^{\mathrm{b}}$ & 160,01 & \pm & $2,42^{\text {defghi }}$ & 556,54 & \pm & $2,19^{\text {hij }}$ \\
\hline PER 002370 & 64,46 & \pm & $0,33^{\text {fgh }}$ & 21,50 & \pm & $0,85^{5^{\mathrm{ijk} \mathrm{l}}}$ & 0,00 & \pm & $0,00^{a a}$ & 165,91 & \pm & $1,38^{\text {cdefg }}$ & 608,15 & \pm & $7,59^{\mathrm{c}}$ \\
\hline PER 002377 & 70,67 & \pm & $0,08^{\mathrm{b}}$ & 25,77 & \pm & $0,85^{\text {def }}$ & 0,82 & \pm & $0,00^{\mathrm{d}}$ & 174,26 & \pm & $2,42^{\text {bcd }}$ & 678,82 & \pm & $9,39^{b}$ \\
\hline PER 002378 & 69,42 & \pm & $0,24^{\mathrm{b}}$ & 19,31 & \pm & $0,01^{\mathrm{lm}}$ & 0,00 & \pm & $0,00^{z}$ & 138,83 & \pm & $4,37^{\mathrm{klm} n}$ & 557,56 & \pm & $13,78^{\mathrm{hij}}$ \\
\hline PER 002379 & 69,34 & \pm & $0,18^{\mathrm{b}}$ & 23,21 & \pm & $0,77^{\text {ghij }}$ & 0,07 & \pm & $0,00^{r}$ & 135,60 & \pm & $3,21^{\mathrm{Imno}}$ & 561,88 & \pm & $5,59^{\text {ghij }}$ \\
\hline PER 002381 & 63,34 & \pm & $0,04^{\mathrm{hi}}$ & 14,69 & \pm & $1,05^{\mathrm{n}}$ & 0,30 & \pm & $0,00^{j}$ & 155,10 & \pm & $2,85^{\text {efghtij }}$ & 604,96 & \pm & $4,74^{\mathrm{e}}$ \\
\hline PER 002380 & 75,69 & \pm & $0,32^{\mathrm{a}}$ & 36,58 & \pm & $0,24^{\mathrm{a}}$ & 0,23 & \pm & $0,00^{\mathrm{m}}$ & 268,20 & \pm & $10,20^{\mathrm{a}}$ & 667,16 & \pm & $2,24^{\mathrm{bc}}$ \\
\hline PER 002422 & 65,93 & \pm & $0,69^{\text {def }}$ & 21,38 & \pm & $0,73^{\mathrm{jkl}}$ & 0,08 & \pm & $0,00^{4}$ & 129,65 & \pm & $2,38^{\text {mop }}$ & 577,57 & \pm & $5,67^{\mathrm{fgh}}$ \\
\hline PER 002442 & 58,98 & \pm & $0,02^{\mathrm{jk}}$ & 25,35 & \pm & $0,85^{\text {deff }}$ & 0,00 & \pm & $0,00^{y}$ & 164,66 & \pm & $3,30^{\text {cdeffg }}$ & 596,12 & \pm & $7,87^{\text {ef }}$ \\
\hline PER 002443 & 59,74 & \pm & $0,57^{\mathrm{j}}$ & 23,61 & \pm & $0,73^{\text {fghi }}$ & 0,00 & \pm & $0,00^{x}$ & 147,15 & \pm & $4,72^{\mathrm{hijkl}}$ & 582,53 & \pm & $5,77^{\mathrm{fg}}$ \\
\hline PER 002456 & 67,46 & \pm & $0,88^{\mathrm{cd}}$ & 19,75 & \pm & $0,77^{\mathrm{klm}}$ & 0,00 & \pm & $0,00^{\mathrm{w}}$ & 181,44 & \pm & $9,77^{\mathrm{b}}$ & 787,14 & \pm & $4,02^{\mathrm{a}}$ \\
\hline PER 002457 & 60,38 & \pm & $0,61^{\mathrm{j}}$ & 21,62 & \pm & $0,83^{\mathrm{ijk}}$ & 0,22 & \pm & $0,00^{\mathrm{n}}$ & 152,59 & \pm & $6,50^{\text {ghijk }}$ & 564,25 & \pm & $4,29^{\text {ghi }}$ \\
\hline PER 002458 & 60,23 & \pm & $0,26^{\mathrm{j}}$ & 22,86 & \pm & $0,84^{\text {hij }}$ & 0,00 & \pm & $0,00^{\mathrm{v}}$ & 157,44 & \pm & $7,99^{\text {efghi }}$ & 596,78 & \pm & $7,23^{\mathrm{ef}}$ \\
\hline PER 002459 & 60,38 & \pm & $0,55^{\mathrm{j}}$ & 23,26 & \pm & $0,83^{\text {ghij }}$ & 0,15 & \pm & $0,00^{\circ}$ & 154,17 & \pm & $7,15^{\text {fyhijk }}$ & 573,93 & \pm & $7,06^{\text {gh }}$ \\
\hline PER 002460 & 52,11 & \pm & $0,36^{\mathrm{no}}$ & 19,37 & \pm & $0,81^{\operatorname{lm}}$ & 0,00 & \pm & $0,00^{u}$ & 135,22 & \pm & $3,10^{\operatorname{lmno}}$ & 500,29 & \pm & $3,98^{\mathrm{m}}$ \\
\hline PER 002461 & 55,51 & \pm & $0,91^{1}$ & 18,05 & \pm & $0,96^{\mathrm{m}}$ & 0,07 & \pm & $0,00^{\mathrm{s}}$ & 141,71 & \pm & $2,30^{\mathrm{jklm}}$ & 549,79 & \pm & $8,36^{\mathrm{ijk}}$ \\
\hline PER 002462 & 66,74 & \pm & $0,66^{\text {cde }}$ & 27,34 & \pm & $0,86^{\text {de }}$ & 0,11 & \pm & $0,00^{\mathrm{p}}$ & 179,91 & \pm & $5,80^{\mathrm{bc}}$ & 790,34 & \pm & $2,18^{\mathrm{a}}$ \\
\hline PER 002463 & 53,22 & \pm & $0,37^{\mathrm{mn}}$ & 17,84 & \pm & $0,86^{\mathrm{m}}$ & 0,26 & \pm & $0,00^{1}$ & 148,57 & \pm & $6,03^{\text {hijkl }}$ & 383,06 & \pm & $22,22^{\mathrm{p}}$ \\
\hline
\end{tabular}

Los valores representan el promedio de tres repeticiones.

La tabla 4 muestra, que la reacción entre los compuestos con capacidad antioxidante y el radical estable 2,2 azinobis (3-etilbenzotiazolina-6-ácido sulfónico) (ABTS), generó valores desde 383,06 a 790,34 $\mu \mathrm{mol}$ TE/100 g bs, pertenecientes a las semillas con código PER 002463 y PER 002462, respectivamente. En investigaciones anteriores se reportan valores ligeramente superiores entre 670,1 a $827,6 \mu \mathrm{mol} \mathrm{TE} / 100 \mathrm{~g}$ de muestra ${ }^{6}$.

\section{CONCLUSIONES}

- Las semillas de kiwicha resaltan por poseer una interesante composición química, de 30 accesiones evaluadas, las semillas con código PER 002380 aportan altos contenidos de proteína $(16,59 \%)$, fibra dietaria $(14,12 \%)$, fenólicos totales $(75,69 \mathrm{GAE} / 100 \mathrm{~g}$ bs) y actividad antioxidante de acuerdo al radical DPPH 268,20 $\mu \mathrm{mol}$ TE/100 g bs. 
- Las semillas de kiwicha tienen un especial aporte de compuestos bioactivos como fibra dietaria, fenólicos totales, flavonoides totales y betalaínas comprendidos entre 7,50 a $14,12 \% ; 51,34$ a $75,69 \mathrm{mg} \mathrm{GAE} / 100 \mathrm{~g}$ bs; 12,65 a $36,58 \mathrm{mg} \mathrm{CE} / 100 \mathrm{~g}$ bs y 0,0 a 1,01 $\mathrm{mg} / 100 \mathrm{~g}$ bs, respectivamente.

\section{AGRADECIMIENTO}

Al Instituto Nacional de Innovación Agraria - INIA del Perú por el financiamiento para el desarrollo de esta investigación en el marco del Proyecto "Evaluación nutricional y compuestos bioactivos de accesiones promisorias de kiwicha del INIA" que se ejecutó en el Área "Valoración y Uso de los Recursos Genéticos" perteneciente a la Subdirección de Recursos Genéticos.

\section{REFERENCIAS BIBLIOGRÁFICAS}

1. AACC International. Method 32-21.01. Insoluble and soluble dietary fibre in oat products enzymatic-gravimetric method. 11th Ed. Saint Paul, Minnesota: Association of American Cereal Chemists; 2010.

2. AOAC. Official Methods of Analysis International. Association of Official Analytical Chemists. Rockville, Maryland: AOAC International; 2016.

3. Barba De La Rosa A, Fomsgaard I, Laursen B, Mortensen A, Olvera L, Silva C, et al. Amaranth (Amaranthus hypochondriacus) as an alternative crop for sustainable food production: Phenolic acids and flavonoids with potential impact on its nutraceutical quality. J Cereal Sci. 2009; 49(1):117-121.

4. Bello-Pérez LA, Agama-Acevedo E, Sánchez-Hernández L, Paredes-López O. Isolation and Partial Characterization of Banana Starches. J Agric Food Chem. 1999; 47(3): 854857.

5. Dini I, Tenore G, Dini A. Antioxidant compound contents and antioxidant activity before and after cooking in sweet and bitter Chenopodium quinoa seeds. LWT - Food Sci Tech. 2010; 43: 447-451.

6. García S, Tironi V. Application of amaranth protein isolate and hydrolysate on a reduced salt fish restructured product: antioxidant properties, textural and microbiological effects. Int J Food Sci Technol. 2015; 50: 1452-1460.

7. Hirose Y, Fujita T, Ishii T, Yueno N. Antioxidative properties and flavonoid composition of Chenopodium quinoa seeds cultivated in Japan. Food Chem; 2010; 119: 1300-1306.

8. Hoover R, Ratnayake W. Determination of Total Amylose Content of Starch. Curr Protocol Food Anal Chem. 2001; 00(1): E2.3.1-E2.3.5.

9. Jain $\mathrm{G}$, Gould K. Are betalain pigments the functional homologues of anthocyanins in plants? Environ Exper Bot. 2015; 119: 48-53.

10. Kanensi O, Ochola S, Gikonyo N, Makokha A. Effect of Steeping and Germination on the Diastatic Activity and Sugar Content in Amaranth Grains and Viscosity of Porridge. J Agric Food Technol. 2013; 3(1): 1-7. 
11. Khan M, Giridhar P. Plant betalains: Chemistry and biochemistry. Phytochem. 2015; 117:267-295.

12. Manach C, Scalbert A, Morand C, Remesy C, Jiménez L. Polyphenols: food sources and bioavailability. Am J Clin Nutr. 2004; 79(5):727-747.

13. Najmus A, Whitney P. Differential behaviour of the dinitrosalicylic acid (DNS) reagent towards mono-and di-saccharide sugars. Biomass Bioenergy. 2011; 35: 4748-4750.

14. Pérez E, Bahnassey Y, Breene W. A Simple Laboratory Scale Method for Isolation of Amaranth Starch. 1993; Starch - Stärke. 1993; 45(6): 211-214.

15. Sené M, Thévenot C, Prioul L. 1997. Simultaneous Spectrophotometric Determination of Amylose and Amylopectin in Starch from Maize Kernel by Multi-wavelength Analysis. J Cereal Sci. 1997; 26: 211-221.

16. Steffensen SK, Rinnan Å, Mortensen AG, Laursen B, de Troiani RM, Noellemeyer EJ, et al. Variations in the polyphenol content of seeds of field grown Amaranthus genotypes. Food Chem. 2011; 129(1):131-138.

17. Re R, Pellegrini N, Proteggente A, Pannala A, Yang M, Rice-Evans C. Antioxidant activity applying and improved ABTS radical cation de colorization assay. Free Radic Biol Med. 1999; 26(9-10): 1231-1237.

18. Repo-Carrasco R, Peña J, Kallio H, Salminen S. Dietary fiber and other functional components in two varieties of crude and extruded amaranth (Amaranthus caudatus). $\mathrm{J}$ Cereal Sci.2009; 49: 219-224.

19. Repo-Carrasco-Valencia RA, Encina CR, Binaghi MJ, Greco CB, Ronayne de Ferrer PA. Effects of roasting and boiling of quinoa, kiwicha and kañiwa on composition and availability of minerals in vitro. J Sci Food Agric. 2010; 90(12):2068-2073.\}

20. Von Elbe J. 2001. Betalains. Curr Protocol Food Anal Chem. 2001; 00(1): F3.1.1F3.1.7. 